\title{
LA PRODUCTION DE LA LAINE ARTIFICIELLE EN ITALIE ET SES RÉPERCUSSIONS SUR L'INDUSTRIE LAITIËRE
}

\author{
par Georges RAY
}

Chef de Service à l'Institut international d'Agriculture de Rome.

On sait combien sont variés les emplois de la caséine, dans l'ordre industriel. Matière plastique de premier ordre, la caséine se prête à la fabrication d'objets moulés (objets de galalite et produits analogues), à la préparation de colles fortes utilisées couramment dans le travail du bois, à la fabrication de peintures et d'enduits solides et économiques, à l'encollage des papiers, au collage des vins et à beaucoup d'autres usages.

A cette liste déjà longue, nous pouvons ajouter aujourd'hui un emploi certainement inattendu : l'utilisation de la caséine comme matière première pour la fabrication de la laine artificielle. Les recherches poursuivies patiemment depuis trois ans sur cette importante transformation industrielle d'un produit agricole (en l'espèce le lait écrémé) par l'Ingénieur Antonio FERRETTI, viennent d'aboutir à la production d'un textile nouveau, appelé par son créateur la laine synthétique (lana sintetica). A mon avis, il serait préférable de dire laine artificielle, en attendant qu'un nom spécial soit trouvé pour le nouveau textile, comme il est advenu pour la soie artificielle, désignée partout maintenant sous le nom de rayonne.

La nouvelle fabrication de la laine artifieielle commence maintenant à entrer dans la phase industrielle. C'est dire que nous devrons attendre un peu avant de pouvoir porter un jugement sur les qualités des tissus de laine de caséine, et l'usage qu'on en peut attendre.

Les premières épreuves expérimentales ont été organisées à Milan, où s'est effectuée la préparation de la caséine, au centre de récolte "Opera» du Consortium des producteurs de lait de Milan. Quant à la transformation de la caséine en "flocons» de la nouvelle fibre textile, elle a été réalisée à l'usine de la Société "Snia Viscosa", à Cesano Maderno.

On ne possède à l'heure actuelle sur la technique de la transformation industrielle de la caséine en laine artificielle que des renseignements d'ordre général, le brevet qui protège l'invention étant pour le moment tenu secret. Toutefois, il est possible de fixer dans ses grandes lignes le mode opératoire adopté, en profitant des déclarations faites il y a peu de jours à la presse par l'Ingénieur Ferretti, déclarations qui ont été reproduites et commentées dans tous les journaux italiens, justement fiers de montrer comment l'Italie réagit à l'application des sanctions.

La matière première utilisée dans la fabrication de la laine 
artificielle est le lait écrémé, tel qu'il sort des écrémeuses centrifuges, c'est-à-dire privé aussi complètement que possible de sa matière grasse (il en reste au plus $0,10 \%$ si l'écrémeuse est bien réglée). On provoque la coagulation du lait écrémé par addition d'une quantité convenable d'acide sulfurique. La caséine est ensuite séparée du sérum, lavée et pressée. On peut alors l'utiliser directement, à l'état humide, ou la conserver par séchage. Quant au sérum, après saturation de son acidité, on peut le destiner à l'alimentation des porcs.

Il faut maintenant passer à la phase de transformation de la caséine en matière textile, ce qui conduit à produire une matière $q u^{\prime}$ on puisse transformer en fils. Iei, l'analogie avee le travail des usines de soie artificielle est évidente.

Pour l'obtention de la laine artifioielle, la caséine doit être traitée par des plastifiants, sur la nature desquels on garde naturellement la plus stricte réserve. On peut cependant résumer comme suit les diverses phases de la fabrication:

$1^{\circ}$ solubilisation de la caséine (probablement par des alcalis ou des sels alcalins),

$2^{\circ}$ maturation du produit,

$3^{\circ}$ passage à la filière, pour l'obtention de fils, rendus solides par trempage dans des bains appropriés,

$4^{\circ}$ séchage.

Le tissage des fils obtenus a permis de présenter des tissus possédant, indépendamment de leur remarquable uniformité, des qualités tout à fait voisines de celles des tissus de laine naturelle, notamment en ce qui concerne l'aspect, la résistance, le pouvoir isolant, l'hygroscopicité, l'aptitude à la teinture. D'ailleurs, le fil de easéine s'apparente, au point de vue de la composition chimique avee la laine naturelle, les deux produits (qui sont d'origine animale) accusant une teneur en azote d'environ $15 \%$.

Au point de vue des rendements, on peut dire qu'il faut mettre en œuvre un poids de caséine sensiblement égal au poids de laine artificielle prête à être tissée. Par rapport au lait écrémé, on compte qu'un hectolitre de lait écrémé donne $3 \mathrm{~kg}$. de laine artificielle prête pour le tissage.

Nous savons maintenant, au moins en gros, comment se présente la question de la fabrication de la laine artificielle. Pour l'Italie; au moment même où sont mises en application les sanctions (dont les conséquences seront redoutables aussi bien pour les pays sanctionnistes que pour l'Italie), la fabrication de la laine artificielle peut contribuer à réduire énormément l'achat des laines naturelles de provenance étrangère. 
Pour les pays autres que l'Italie, et notamment pour ceux qui sont à la recherche de débouchés nouveaux pour une partie de leur production laitière, il est bien évident que le procédé de fabrieation dela laine de caséine présente un intérêt économique de premier ordre.

Enfin, les pays producteurs et exportateurs de grandes quantités de laine naturelle peuvent se demander non sans inquiétude si leurs exportations ne sont pas destinées à décroître dangereusement dans un avenir assez prochain.

Bien d'autres incidences de l'apparition de la laine de caséine seraient à considérer et à discuter. En ce qui concerne le eôté purement industriel, il est fort probable que le nouveau textile inventé par l'Ingénieur Ferretti prendra rapidement dans le marché mondial une place aussi importante que celle actuellement occupée par la rayonne, et que d'innombrables tissus, de laine artificielle seule ou de laine artificielle mélangée à d'autres textiles (tissus mixtes), seront offerts au public dans un prochain avenir. Ceci ne veut pas dire, bien entendu, que la fabrication des tissus de laine naturelle soit fatalement compromise, mais elle devra s'organiser en tenant compte de la situation nouvelle eréée par l'apparition de la laine de oaséine sur le marché. Par beaucoup de points, cette situation s'apparentera à la lutte à laquelle nous assistons depuis une vingtaine d'années entre la soie naturelle et les soies artificielles obtenues par des procédés très variés de transformation d'une matière première commune, la cellulose. Mais nous nous adressons à des agriculteurs, et nous ne voulons retenir l'attention que sur les aspects spécifiquement agricoles du problème qui nous occupe ici.

En examinant sommairement les répercussions que provoquera fatalement sur l'économie générale de l'Italie la création d'une importante industrie de transformation de la caséine en laine artificielle, on s'aperçoit que les conditions de production de la laine naturelle, du lait et des produits dérivés (beurres et fromages) seront eonsidérablement modifiées, pour ne pas dire bouleversées de fond en comble.

L'an dernier (1934), les importations de laine en Italie ont atteint les chiffres suivants:

\begin{tabular}{|c|c|c|c|c|}
\hline $\begin{array}{l}\text { Laine brute. } \\
\text { Laine lavée. }\end{array}$ & $\begin{array}{r}588.450 \\
79.261\end{array}$ & quintaux, & vala & $\begin{array}{r}394.836 .370 \\
73.637 .580\end{array}$ \\
\hline échets de laine & 44.556 & y & " & 42.586 .300 \\
\hline Total & 712.267 & n & » & 511.060 .250 \\
\hline
\end{tabular}

Les techniciens italiens estiment, pour le moment, qu'en tenant compte des disponibilités intérieures en laine naturelle (12 à 13 millions de $\mathrm{kg}$. dont le $1 / 4$ au moins sert à la fabrication des matelas et des couvertures), et de la modeste exportation de laine brute et 
de tissus de laine, les besoins de l'Italie s'élèvent annuellement à une trentaine de millions de $\mathrm{kg}$. de laine lavée. Comme la production nationale de laine naturelle n'est pas susceptible d'augmentation notable et rapide, il faudrait done, pour supprimer en grande partie l'importation de la laine naturelle, fabriquer, à partir de la caséine, 30 millions de $\mathrm{kg}$. de laine artificielle, ce qui suppose la mise en œuvre de 10 millions d'hectolitres de lait écrémé.

Or actuellement, les disponibilités de lait écrémé en Italie ne dépassent pas 3 à 4 millions d'hectolitres. En effet, si la production beurrière de l'Italie atteint annuellement 450 à 500.000 quintaux, un tiers seulement de cette quantité provient de l'écrémage direct, les deux autres tiers étant fournis par l'industrie fromagère.

De plus, jusqu'à présent, l'industrie de la caséine n'a pas dépassé une productivité de 25 à 30.000 quintaux. Les besoins nouveaux résultant de la fabrication de la laine artificielle ne pourraient être satisfaits que par un accroissement considérable du nombre des vaches laitières, et par l'organisation de centres de ramassage du eaillé, une saine économie conduisant à utiliser la caséine fraîche, pour éviter les frais de séchage.

En tablant sur une production moyenne annuelle de 1.500 litres de lait par vache, il faudrait, pour satisfaire aux besoins en matière première de la nouvelle industrie (environ 7 millions d'hectolitres de lait en plus par an), recruter et nourrir plus de 450.000 vaches. Or le recensement de 1930 accusait la présence de 2.388 .000 vaches en Italie. Ce nombre devrait être rapidement augmenté à peu près d'un cinquième !

Poursuivons maintenant notre argumentation. A la production d'un supplément de 7 millions d'hectolitres de lait correspondrait l'obtention d'une masse de beurre de 30 millions de $\mathrm{kg}$. qu'on devrait utiliser en grande partie sur place, l'exportation du beurre n'étant pas assurée actuellement d'un débouché facile. Or, si l'on transforme difficilement du jour au lendemain les habitudes alimentaires d'un pays, e'est un fait que la consommation de beurre en Italie est actuellement faible, et qu'elle pourrait être notablement accrue, mais ce serait bien entendu aux dépens des autres graisses alimentaires, dont la consommation devrait être restreinte ou interdite.

On voit combien sont sérieuses les répercussions d'une nouvelle industrie du lait sur l'ensemble de l'économie d'un pays. Et nous n'avons volontairement envisagé, pour abréger notre exposé, que les incidences principales du problème.

Quoiqu'il en soit, ce problème est maintenant posé de la façon la plus nette pour l'Italie, pays où la fabrication industrielle de la laine de caséine sera prochainement un fait accompli. Il n'est pas besoin d'être grand clere pour prévoir que la fabrication de la laine 
de easéine ne se cantonnera pas très longtemps en Italie, et qu'elle sera tentée également dans les autres pays laitiers, quelle que soit d'ailleurs la réaction de l'industrie de la laine naturelle.

Il m'a semblé en tous cas extrêmement important que les agriculteurs français soient mis sans retard au courant d'une découverte appelée à transformer profondément l'économie des pays grands producteurs de lait.

Comme il serait vain de songer à arrêter ou à retarder le perfectionnement de la technique industrielle, il convient dès à présent d'étudier dans son ensemble fort complexe, le problème essentiellement nouveau dont je n'ai fait qu'indiquer sommairement les aspects les plus caractéristiques. Je me propose d'ailleurs de compléter ces informations, dès que cela sera possible, mais il n'est pas nécessaire d'attendre pour saluer comme il convient l'apparition d'une nouvelle et très importante industrie agricole, dont le créateur mérite les plus vifs éloges.

\section{BIBLIOGRAPHIE ANALYTIQUE}

\section{LES LIVRES}

\section{Burke (A. D.). - Practical ice Cream making and practical}

mix tables (Guide pratique de fabrication de la crème glacée). Un volume relié toile, 212 pages, tableaux et schémas. - Editeur: The Olsen Publishing $\mathrm{C}^{\circ}, 505$, West Cherry Street à Milwaukee (Wis. U. S. A.), 1933. Prix : 2 dollars.

La littérature américaine est déjà riche en ouvrages traitant de l'industrie de la crème glacée, industrie si florissante aux Etats-Unis.

Voici un nouvel ouvrage dû à la compétence du professeur A. D. BuRKE, ouvrage particulièrement chargé en données essentielles pratiques, comprenant entre autres de nombreuses formules de " mélanges".

Après un rapide historique concernant la crème glacée, et un rappel des caractéristiques de la constitution du lait, B. traite successivement :

$1^{0}$ Los produits laitiers utilisés pour les mélanges (matière grasse, poudre de lait, lait condensé, lait concentré, ete.).

$2^{\circ}$ Standardisation du lait, de la crème et de la crème glacée (tablesstandards à l'usage des industriels).

$3^{0}$ Standardisation des mélanges. - Chapitres comprenant après des considérations théoriques sur les méthodes employées et les buts poursuivis, l'établissemənt de solutions pratiques aux différents problèmes posés (mélangas simples et mélanges complexes).

4. Fabrication proprement dite (calcul des poids des composants tables indicatrices de formules).

$5^{\circ}$ Introduction des différents parfums (crème glacée au chocolat, aux fruits, emploi du miel et autres substances. 